\title{
Ge immersed grating manufacturing and optical verification for the METIS high-resolution spectrograph
}

Agócs, Tibor, Elswijk, Eddy, Zaalberg, Daan, Peterzon, Jan Rinze, Tromp, Niels, et al.

Tibor Agócs, Eddy Elswijk, Daan Zaalberg, Jan Rinze Peterzon, Niels Tromp, Ivan Lloro, Jeffrey Lynn, Ramon Navarro, Takashi Sukegawa, Yukinobu Okura, Stephen Todd, Alistair Glasse, Philip Parr-Burman, Bernhard Brandl, Felix Bettonvil, "Ge immersed grating manufacturing and optical verification for the METIS high-resolution spectrograph," Proc. SPIE 11451, Advances in Optical and Mechanical Technologies for Telescopes and Instrumentation IV, 114511G (13 December 2020); doi: 10.1117/12.2562411

SPIE. Event: SPIE Astronomical Telescopes + Instrumentation, 2020, Online Only 


\title{
Ge immersed grating manufacturing and optical verification for the METIS high-resolution spectrograph
}

\author{
Tibor Agócs*a , Eddy Elswijkª, Daan Zaalberga, Jan Rinze Peterzon ${ }^{\mathrm{a}}$, Niels Tromp ${ }^{\mathrm{a}}$, Ivan Lloro ${ }^{\mathrm{a}}$, \\ Jeffrey Lynn ${ }^{\mathrm{a}}$, Ramon Navarro ${ }^{\mathrm{a}}$, Takashi Sukegawa ${ }^{\mathrm{b}}$, Yukinobu Okura ${ }^{\mathrm{b}}$, Stephen Todd ${ }^{\mathrm{c}}$, Alistair \\ Glasse $^{\mathrm{c}}$, Phil Parr-Burman ${ }^{\mathrm{c}}$, Bernhard Brandl ${ }^{\mathrm{d}, \mathrm{e}}$, Felix Bettonvil ${ }^{\mathrm{a}, \mathrm{d}}$ \\ aNOVA Optical Infrared Instrumentation Group at ASTRON, Oude Hoogeveensedijk 4, 7991 PD \\ Dwingeloo, The Netherlands; ${ }^{b}$ Canon Inc., 30-2, Shimomaruko 3-chome, Ohta-ku, Tokyo 146-8501, \\ Japan; 'UK Astronomy Technology Centre, Edinburgh EH9 3HJ, UK; ' Leiden Observatory, Leiden \\ University, P.O. Box 9513, 2300 RA Leiden, The Netherlands; 'Faculty of Aerospace Engineering, \\ Delft University of Technology, Kluyverweg 1, 2629 HS Delft, The Netherlands
}

\begin{abstract}
We present the manufacturing and optical verification of the germanium immersed grating for the $\mathrm{L} / \mathrm{M}$ band high resolution spectrograph (LMS). The LMS is one of the science subsystems of the Mid-infrared ELT Imager and Spectrograph, METIS. The immersed grating has very demanding requirement specifications: $<100 \mathrm{~nm}$ RMS transmitted Wave Front Error (WFE) after double pass, and $>70 \%$ peak throughput in all orders within the 2.9-5.3 $\mu$ m wavelength range over the pupil. The grating has a period of $18.2 \mu \mathrm{m}$, a sawtooth groove profile with 89.6 degrees apex angle and a grating area of $150 \mathrm{~mm} \times 60 \mathrm{~mm}$. The germanium immersed grating was produced by Canon's high precision mechanical cutting technology. We present the interferometric tests that were performed in order to verify WFE and two different measurements (based on cascade laser and Fourier Transform Spectrometer, respectively) for throughput verification.
\end{abstract}

Keywords: Immersed grating, germanium, METIS, mid-infrared, manufacturing, verification, wavefront error, throughput

\section{INTRODUCTION}

METIS, the Mid-infrared E-ELT Imager and Spectrograph, covering the L, M and $\mathrm{N}$ band atmospheric windows, will offer imaging, coronagraphy and low/medium-resolution spectroscopy over the full wavelength range (2.9-13.5 $\mu \mathrm{m})$, and high-resolution integral field spectroscopy in L (2.9-4.2 $\mu \mathrm{m})$ and $\mathrm{M}(4.5-5.2 \mu \mathrm{m})$ bands. Amongst others, it will address science questions in the area of proto-planetary disks, formation of planets, exoplanet detection and characterization. Figure 1 shows METIS on the Nasmyth platform and the mechanical layout inside the cryostat.

METIS consists of two diffraction-limited imagers [1], IMG-LM and IMG-N, operating in the LM and $\mathrm{N}$ bands, respectively and an Integral Field Unit (IFU) fed diffraction-limited high-resolution ( $\mathrm{R}=100,000)$ LM band Spectrograph (LMS). It also provides focal plane and pupil plane coronagraphy for all operating modes to achieve high contrast imaging [2]. The Common Fore Optics (CFO) provides the opto-mechanical interfaces between the various subsystems and is responsible for chopping, image de-rotation, pupil stabilization, thermal background and stray light reduction [3]. All these subsystems, together with an in-built infrared wavefront sensor (SCAO, Single Conjugate Adaptive Optics system), are located in the cryostat. The Warm Calibration Unit (WCU) is responsible for routine daily daytime calibrations and alignment verification of METIS [4] during the assembly, integration and verification (AIV) phase. The METIS cryostat and the WCU are held in position by the Warm Support Structure (WSS). The optical overview of METIS is shown in Figure 2.

*agocs@astron.nl; phone +31 521595173

Advances in Optical and Mechanical Technologies for Telescopes and Instrumentation IV, edited by Ramón Navarro, Roland Geyl, Proc. of SPIE Vol. 11451,114511G

(C) 2020 SPIE · CCC code: $0277-786 X / 20 / \$ 21 \cdot$ doi: $10.1117 / 12.2562411$ 

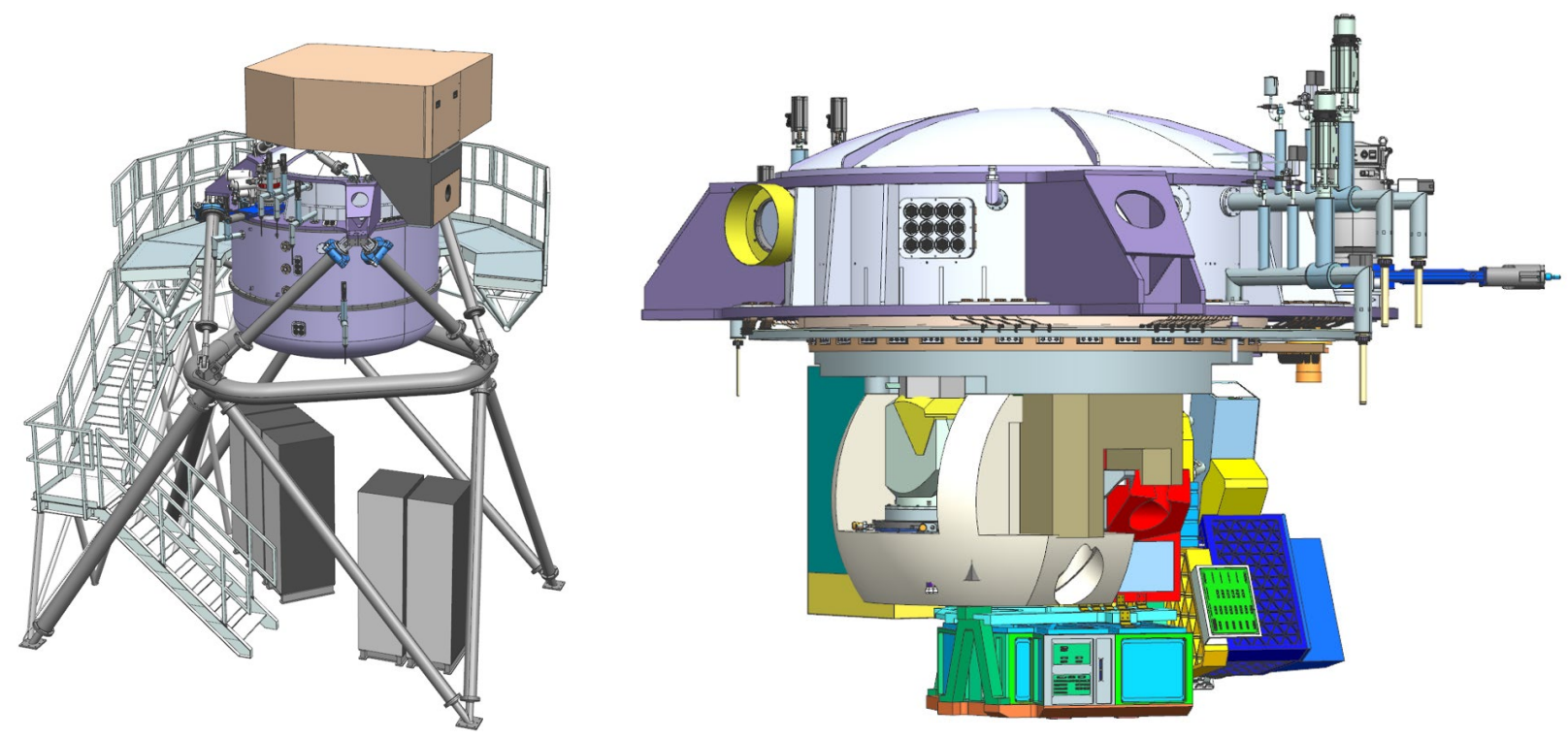

Figure 1. METIS on the Nasmyth platform of the E-ELT (left). METIS mechanical layout inside the cryostat (right).

The aim of the paper is to the present the Ge immersed grating manufacturing and optical verification for the LMS. In section 2, we present the IFU fed LMS and its key component, the Ge immersed grating in detail. In section 3, we present the manufacturing method used to produce the Ge immersed grating. Afterward, we present the optical configurations and discuss the obtained results from the optical verification of the grating, related to the wavefront error (section 4) and throughput (section 5). In section 6, we summarize the achievements and outline the next steps regarding the grating verification process.

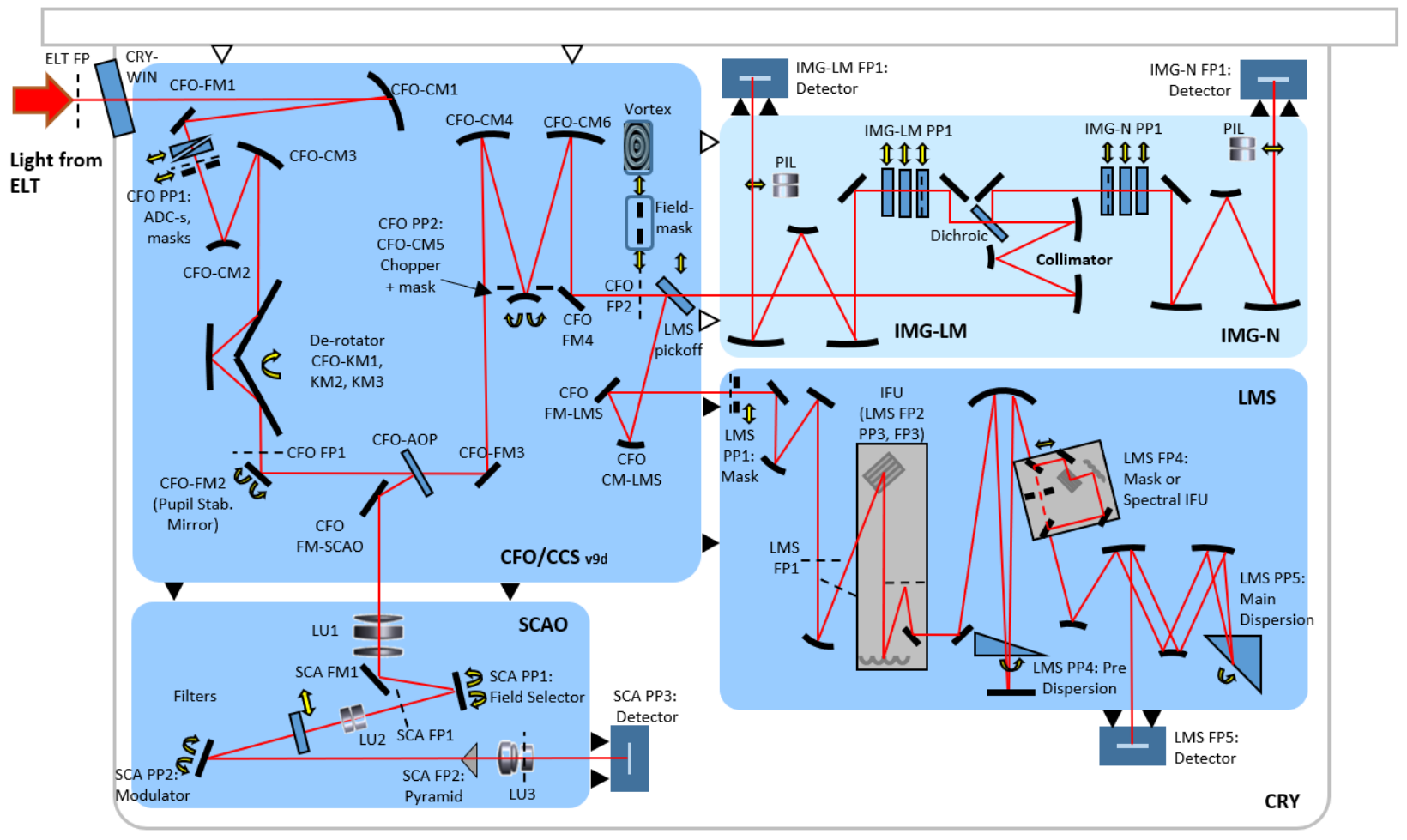

Figure 2. The optical overview of the METIS cryostat is shown. 


\section{LMS AND THE GE IMMERSED GRATING}

The LMS provides high resolution $(\mathrm{R} \sim 100,000)$ spectra over a small band in the range 2.7-5.3 $\mu \mathrm{m}$. An image slicing integral field unit (IFU) provides spatially resolved spectra. An extended wavelength coverage mode $(\Delta \lambda \geq 300 \mathrm{~nm})$ is provided by a 'spectral slicing' system which allows multiple diffraction orders of the echelle to be observed simultaneously, at the expense of a reduced field of view on sky.

The optical interface between the CFO and the LMS is the pupil with a relatively small 3 arcsec field of view. A mask exchange wheel will be included at the input pupil plane to allow for different pupil aperture masks and other components to be deployed for observing modes including high contrast imaging modes. Subsequently, the transfer optics creates a magnified image of the focal plane on the slicing mirror of the IFU. The IFU is an all-reflective image slicing integral field unit. The magnified image produced by the transfer optics is sliced, reformatted and re-imaged to produce the input slit for the pre-disperser. The input FOV of the IFU is $1.00 \times 0.58 \operatorname{arcsec}^{2}$ and the slice width is matched to $\lambda / \mathrm{D}$ at $\lambda=3.7 \mu \mathrm{m}$, giving 20.7 mas on-sky. The IFU is followed by the pre-disperser, which is a low resolution prism spectrometer with an exit slit mask. This acts as a low spectral resolution monochromator, defining the bandpass within the LM band, acting as an order sorting filter for the echelle. The main disperser is a high resolution echelle spectrometer. The dispersing element is a germanium immersed grating. A three mirror system is used in double pass to form the collimator and camera of the spectrometer. The instantaneous coverage of the spectrum is very small compared to the complete LM band. Multiple exposures with rotation of the predisperser and main disperser are required to build up a continuous spectrum.
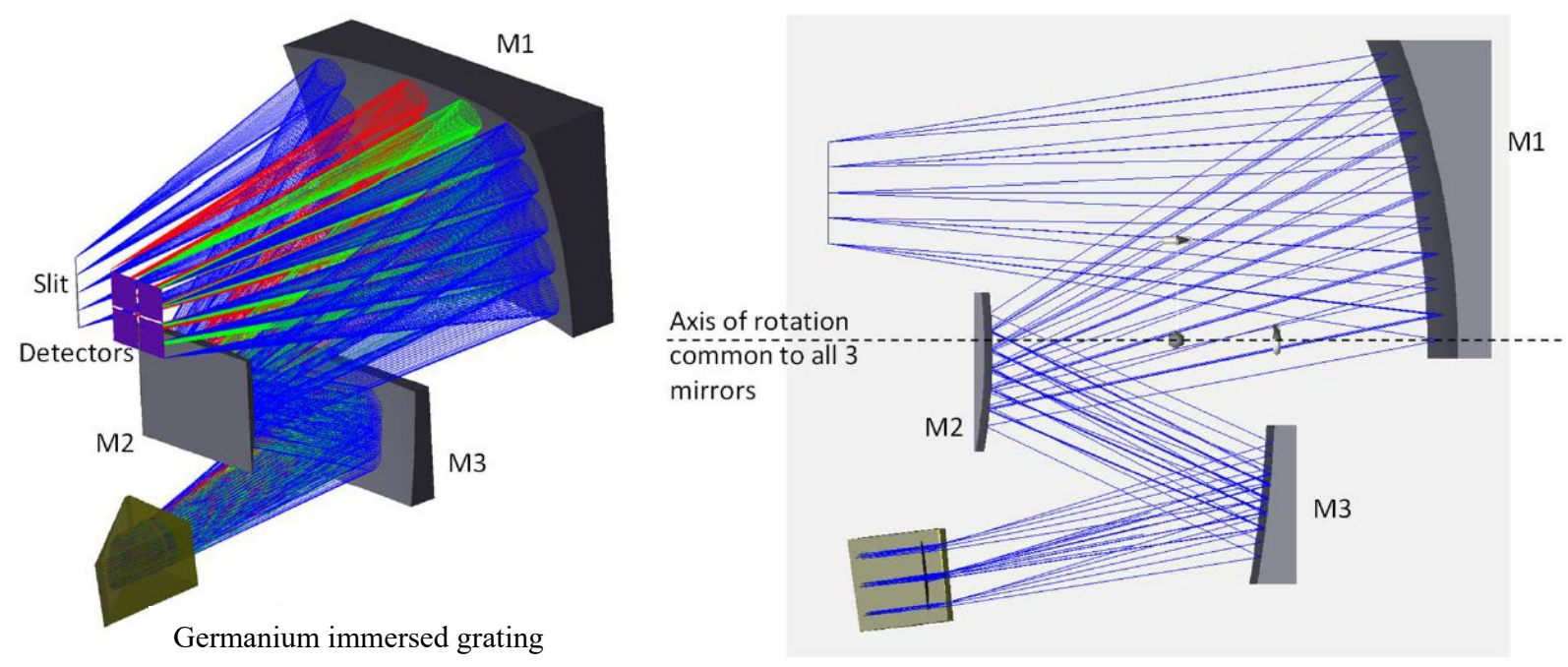

Figure 3. Two views of the main disperser assembly of the LM band Spectrograph are shown.

The immersed grating (Figure 4), acting as the main dispersing element in the LMS, increases the optical path difference and angular dispersion proportionally to the refractive index $(n=4)$. Consequently, a smaller grating area is sufficient to obtain the same spectral resolution, and a significant reduction of spectrometer volume becomes possible. The immersed grating has very demanding requirement specifications: $<100 \mathrm{~nm}$ RMS transmitted WFE after double pass, and $>70 \%$ peak throughput over the pupil. The grating has a period of $18.2 \mu \mathrm{m}$, a sawtooth groove profile with 89.6 degrees apex angle and a grating area of $150 \times 60 \mathrm{~mm}^{2}$.

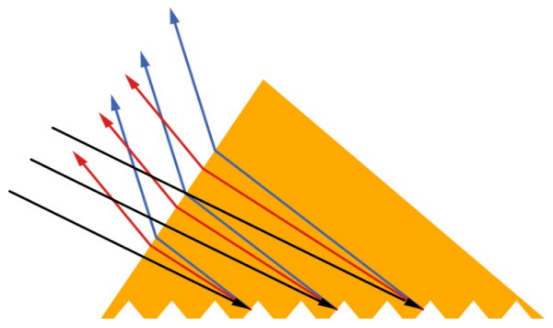

Figure 4. The immersed grating principle is shown: light enters the prism through a polished entrance facet; after diffraction at the grating facet inside the material, the dispersed light leaves the prism through the same facet. 


\section{MANUFACTURING}

The germanium immersed grating was produced by Canon's high precision mechanical cutting technology. Single crystal germanium is used as the substrate for the immersed grating. First, the prism shape is manufactured to specifications and then the prism is processed by fly-cutting to generate the grating pattern. Fly-cutting is a common cutting method, where the diamond tool rotates at high speed, while the substrate is translated in one direction. Figure 5 shows the machining setup and movement. The diamond tool orientation is approximately perpendicular to the tool travel direction, and the groove is formed in the tool travel direction. Since Ge is hard and brittle, it is necessary to make a very small amount of cut in one revolution compared with general metals, but good surface roughness of 3nm RMS or less can be obtained.

A single groove is formed by cutting and removing germanium by rotation and linear motion using the diamond tool with 90 degree blade edge according to the grating design. The tool is incremented by the grating pitch after cutting each groove. Since the diamond tool does not interfere with the existing groove during processing, it is sufficient to use a diamond tool size slightly larger than the pitch.
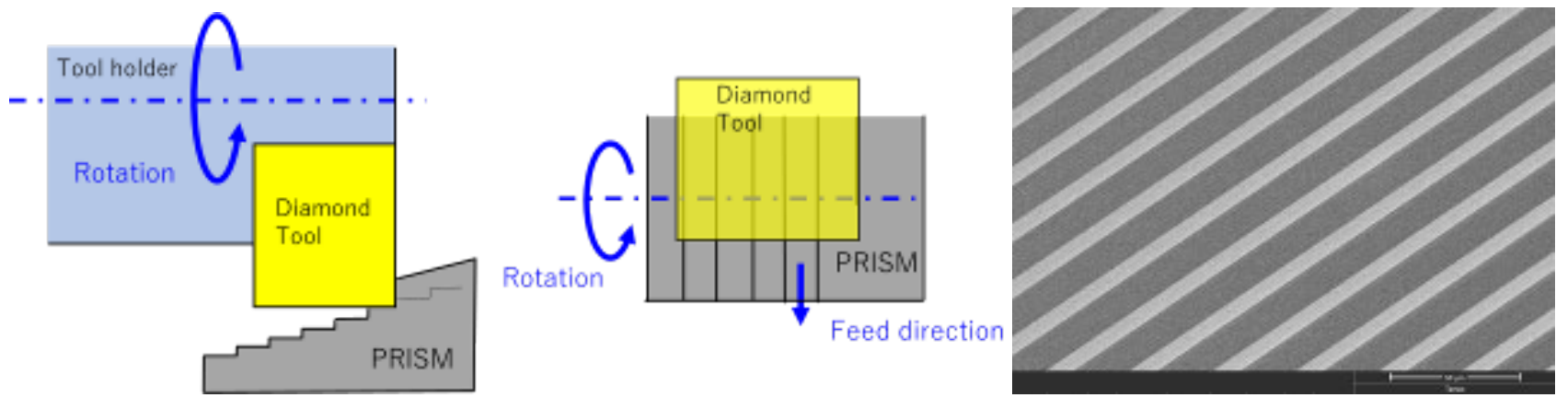

Figure 5. The fly-cutting machining setup and movement are shown (left, middle). The SEM photograph of 18.2 um pitch germanium grating is shown on the right.

Since material deformation is very small in brittle material processing, even a slight tool vibration affects the manufacturing process and the desired ridgeline cannot be obtained. From the manufacturing's point of view, longer grating pitch is favorable, which generally suitable for thermal infrared wavelength around $10 \mu \mathrm{m}$. The METIS immersed grating has a grating pitch of $18.2 \mu \mathrm{m}$, which is on the short side, meaning more challenging processing and higher risk of defective grating surface. The SEM photograph of the 18.2 um pitch germanium grooves is shown in Figure 5.
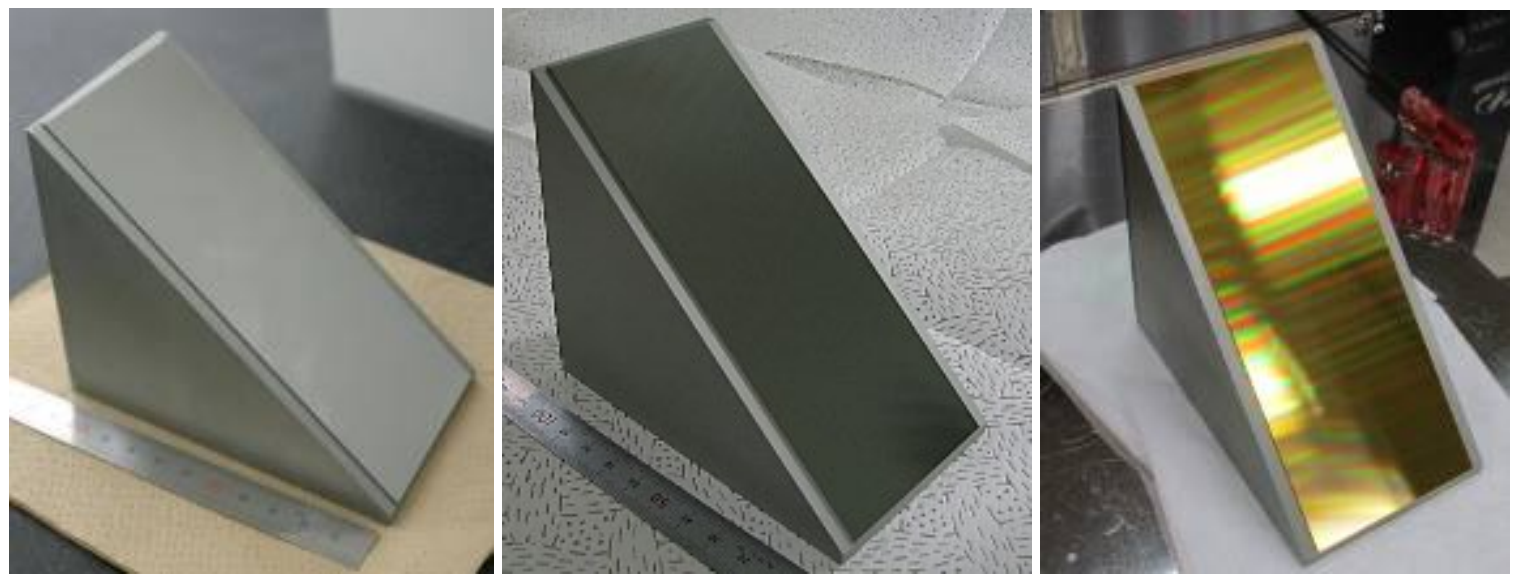

Figure 6. METIS immersed grating is shown before (left) and after (middle) machining, and after gold coating (right).

The germanium has a very high refractive index, so anti-reflection coating is necessary on the entrance/exit surface in order to prevent a significant light-loss due to surface Fresnel losses. The antireflection coating is a multilayer film optimized for the $2.9 \mu \mathrm{m}$ to $5.3 \mu \mathrm{m}$ wavelength range. A gold back reflection coating is applied to the grooved surface. In Figure 6 the METIS immersed grating is shown before and after machining, and after gold coating. More information on Canon's capabilities related to germanium immersed gratings for astronomy can be found in [5]. 


\section{WAVEFRONT ERROR MEASUREMENTS}

Interferometric tests were done with a Zygo HeNe laser interferometer, operating at $633 \mathrm{~nm}$, to measure the entrance/exit facet, as well as the grating surface from air-side using Littrow configuration. The immersed grating has very demanding requirement specifications: $<100 \mathrm{~nm}$ RMS transmitted WFE after double pass.

In the following images the interferometric measurements are shown after masking the useful area of the entrance/exit surface as well as the grating surface. The entrance/exit surface was measured perpendicularly to the surface, while the grating surface was measured under 40 degrees that corresponds to the Littrow configuration for the air-side measurement with the visible HeNe laser.
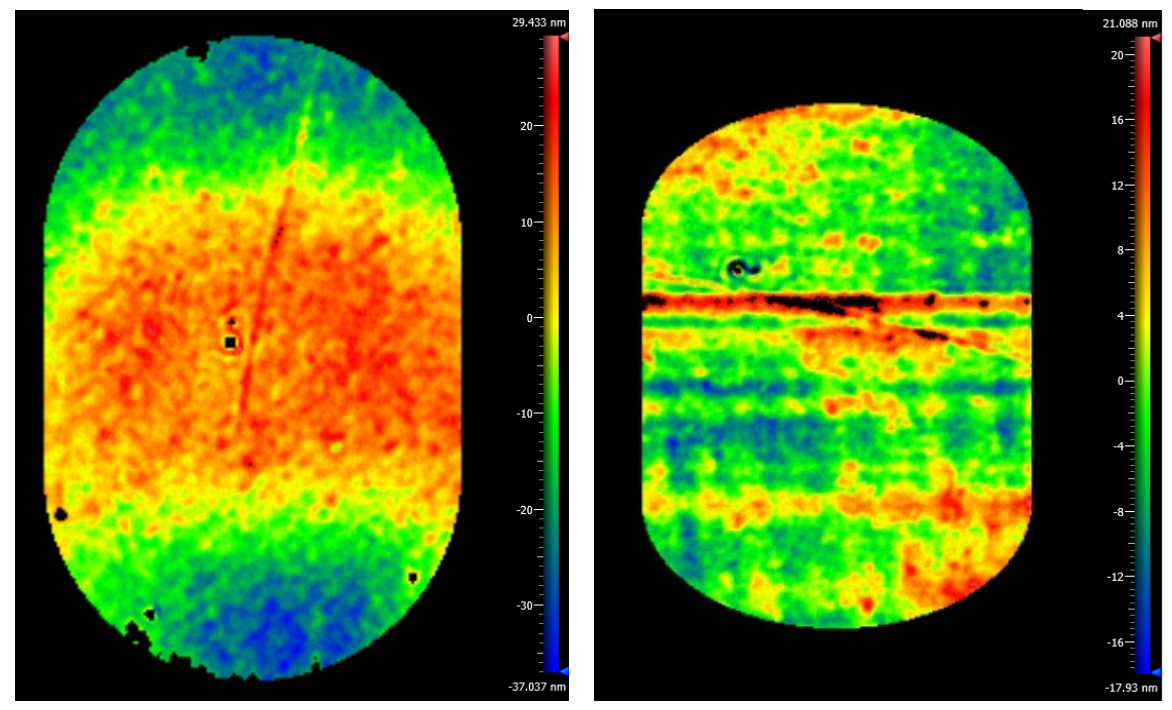

Figure 7. Interferometric images are shown for the $85 \mathrm{~mm}$ x $55 \mathrm{~mm}$ entrance/exit surface (left) and the $115 \mathrm{~mm}$ x $55 \mathrm{~mm}$ grating surface (right) for the useful areas (clear apertures). The latter appears smaller due to the 40 degree angle of observation. The corresponding RMS surface form error are $14.1 \mathrm{~nm}$ and $6.1 \mathrm{~nm}$, respectively.

The measured RMS WFE of the entrance/exit surface is $14.1 \mathrm{~nm}$ after subtracting piston, tip and tilt. It is measured over the complete clear aperture of the surface $(85 \mathrm{~mm}$ x $55 \mathrm{~mm})$. The grating surface is even flatter than the entrance/exit surface, but has some distinct linear features parallel to the rulings (visible in the upper half of the aperture). The clear aperture (115 mm x $55 \mathrm{~mm}$ ) on this surface is scaled assuming an angle of incidence of $40 \mathrm{deg}$ (that corresponds to the Littrow configuration).

The overall double pass WFE of the immersion grating can be calculated by summing up the measured WFE by the various contributors: \#1: prism front surface and \#2: grating WFE reflected at air side. Regarding \#1, the incoming and returning beams do not fully overlap, therefore these two air-surface interfaces are treated as semi-independent $(\sqrt{ } 2)$. On the immersed side the grating WFE (\#2) has an effect that is the index of refraction times higher than on the air side of the grating. The WFE formula from these individual contributions is thus:

$$
W F E_{I G}=\sqrt{[\sqrt{2}(n-1) \cdot \# 1]^{2}+[n \cdot \# 2]^{2}}
$$

, where $\mathrm{n}$ is the index of refraction of germanium $(\mathrm{n}=4)$.

The formula gives an overall double pass WFE of $64.6 \mathrm{~nm}$ RMS, which is below the $100 \mathrm{~nm}$ RMS specification. It probably even has some margin due to the fact that at the entrance/exit surface the footprints of the input and output beams are smaller than the complete clear aperture. On the other hand, the calculation does not take into account the material related effects (birefringence, refractive index inhomogeneity), but they considered to be significantly lower than the surface form errors due to the single crystal germanium substrate used. More information on these effects can be found in [6]. 


\section{THROUGHPUT MEASUREMENTS}

In the present section we outline the optical arrangements and discuss the results of the TE/TM throughput measurements. They were first performed with a tunable cascade laser at $4 \mu \mathrm{m}$ wavelength at Canon's facilities in Japan. Subsequently, the throughput measurements were repeated using a modified Fourier Transform Spectrometer in order to provide a broader wavelength coverage at NOVA in the Netherlands. According to the requirement specification, the grating shall achieve $>70 \%$ peak throughput in all orders within the $2.9-5.3 \mu \mathrm{m}$ wavelength range over the pupil.

\section{Cascade laser TE/TM throughput measurement (around $4 \mu \mathrm{m}$ )}

The throughput of the germanium grating was measured around $4 \mu \mathrm{m}$ using the configuration depicted in Figure 8 . A MIRcat tunable cascade laser from Daylight Solutions (U.S.A) was used. The laser wavelength could be tuned to be between $3.74 \mu \mathrm{m}$ and $4.14 \mu \mathrm{m}$. The bandwidth of this laser was narrower than the theoretical spectral resolution of the fabricated immersion grating. The setup contained a reference arm to monitor and calibrate laser source fluctuations. The immersed grating throughput measurements were referenced to a gold mirror, which was measured independently.

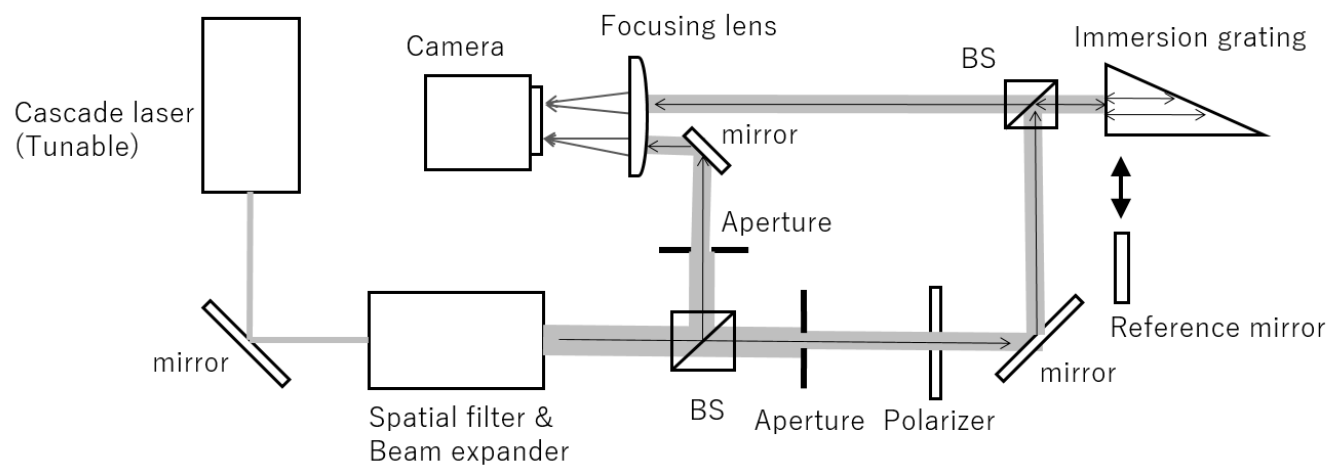

Figure 8 . The tunable cascade laser based configuration used to measure grating throughput (around $4 \mu \mathrm{m}$ ) .

The measurement results are shown in Figure 9. For TE polarization, the peak throughput is $\sim 78 \%$ and for TM polarization it is significantly lower, below $60 \%$. For the latter three neighboring diffraction orders are measured and at the crossover between orders the diffraction efficiency is $\sim 32 \%$. The reason for this throughput difference between the throughput for TM and TE input polarization is due to the facet design and grating geometry and it is expected according to the diffraction efficiency modeling of the grating.

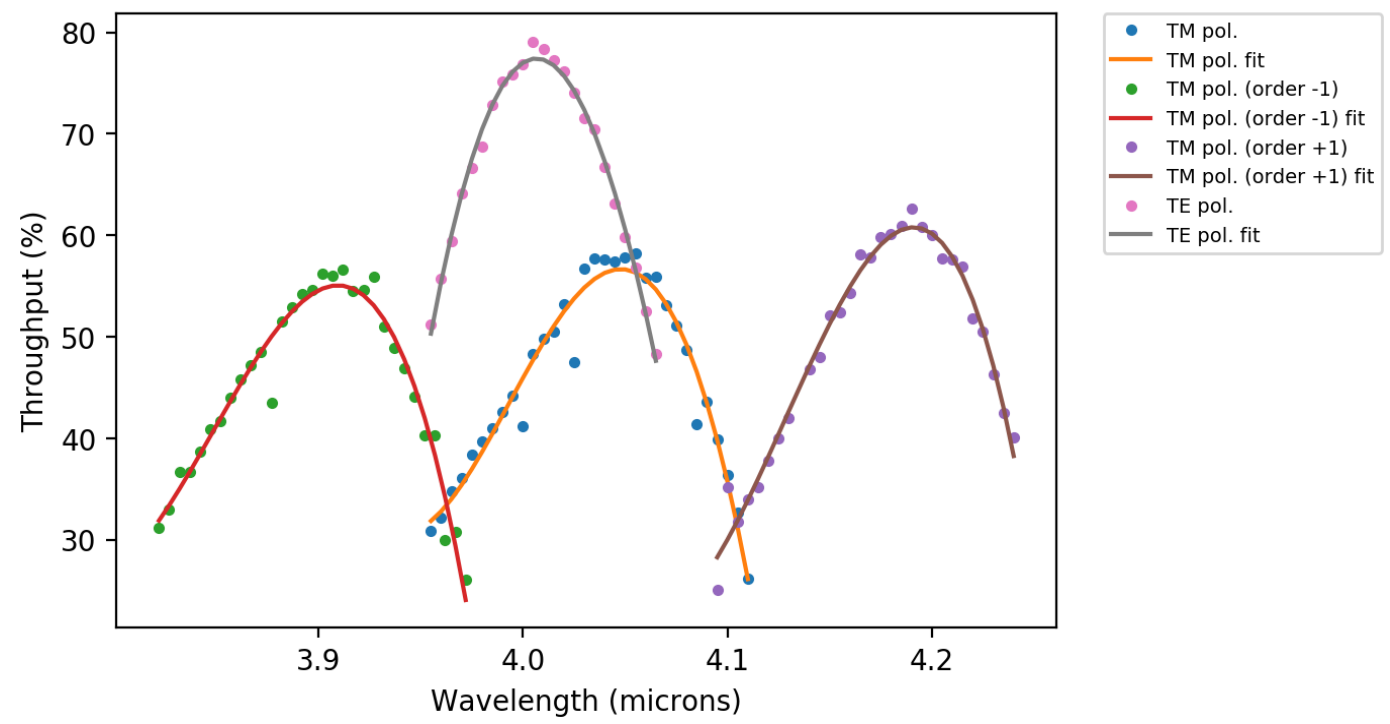

Figure 9. Throughput measurement results from the MIRcat cascade laser based measurements. 


\section{FTS based TE/TM throughput measurements $(2.9-5.3 \mu \mathrm{m})$}

The throughput was also measured by a Varian 7000e FT-IR Spectrometer (FTS) at the NOVA Optical-Infrared group in Dwingeloo (NL). The wavelength range of the FTS ranges from $200 \mathrm{~nm}$ to $28 \mu \mathrm{m}$ and covers the entire METIS wavelength range $(2.9-5.3 \mu \mathrm{m})$. The raw data from the FTS measurement was normalized by the reference (uncoated gold mirror) reflectivity to obtain the absolute throughput of the IG. In the wavelength range of interest a freshly coated gold coating provides $99 \%$ reflectivity, for the normalization factor we used a more realistic reflectivity of $98 \%$.

A dedicated FTS setup was designed and built in order to be able to measure the throughput of the IG in a scenario that resembles the METIS environment the best. Normally, in the sample compartment, light is focused on a small spot and the sample can be measured in transmission. We designed a special relay system, based on a small roof-top mirror (placed in the vicinity of the focus) coupling the light in/out and an off-axis paraboloid mirror collimating the IR light (up to $60 \mathrm{~mm}$ diameter), which can be used to measure the sample in reflection.
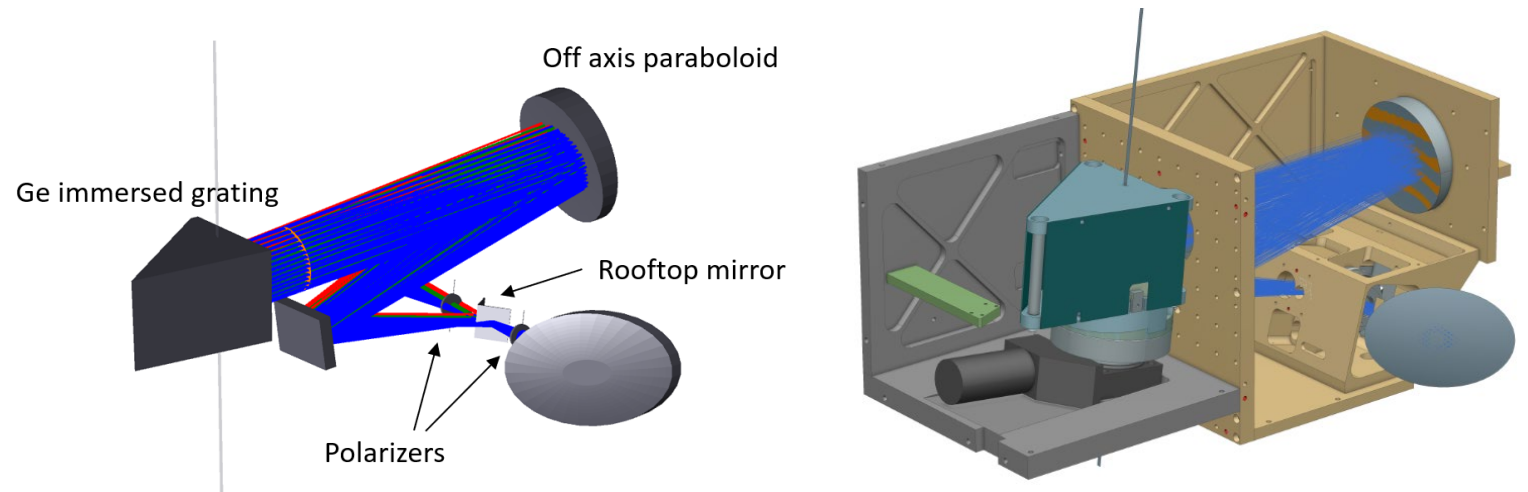

Figure 10. A dedicated FTS setup was designed and built at NOVA-ASTRON in order to measure the throughput Ge immersed grating. The OpticStudio ${ }^{\circledR}$ model (left) and the mechanical CAD model (right) are shown.

The Ge immersed grating was placed on a motorized rotation stage to be able to scan the complete wavelength range of interest. In the relay optics, before the rooftop mirror in the input beam, a linear (wire-grid) polarizer could be placed. It was mounted on a SmarAct motorized rotation stage. Both the immersed grating and linear polarizer rotation could be controlled thorugh a dedicated computer to allow uninterrupted measurement sequences and automation.
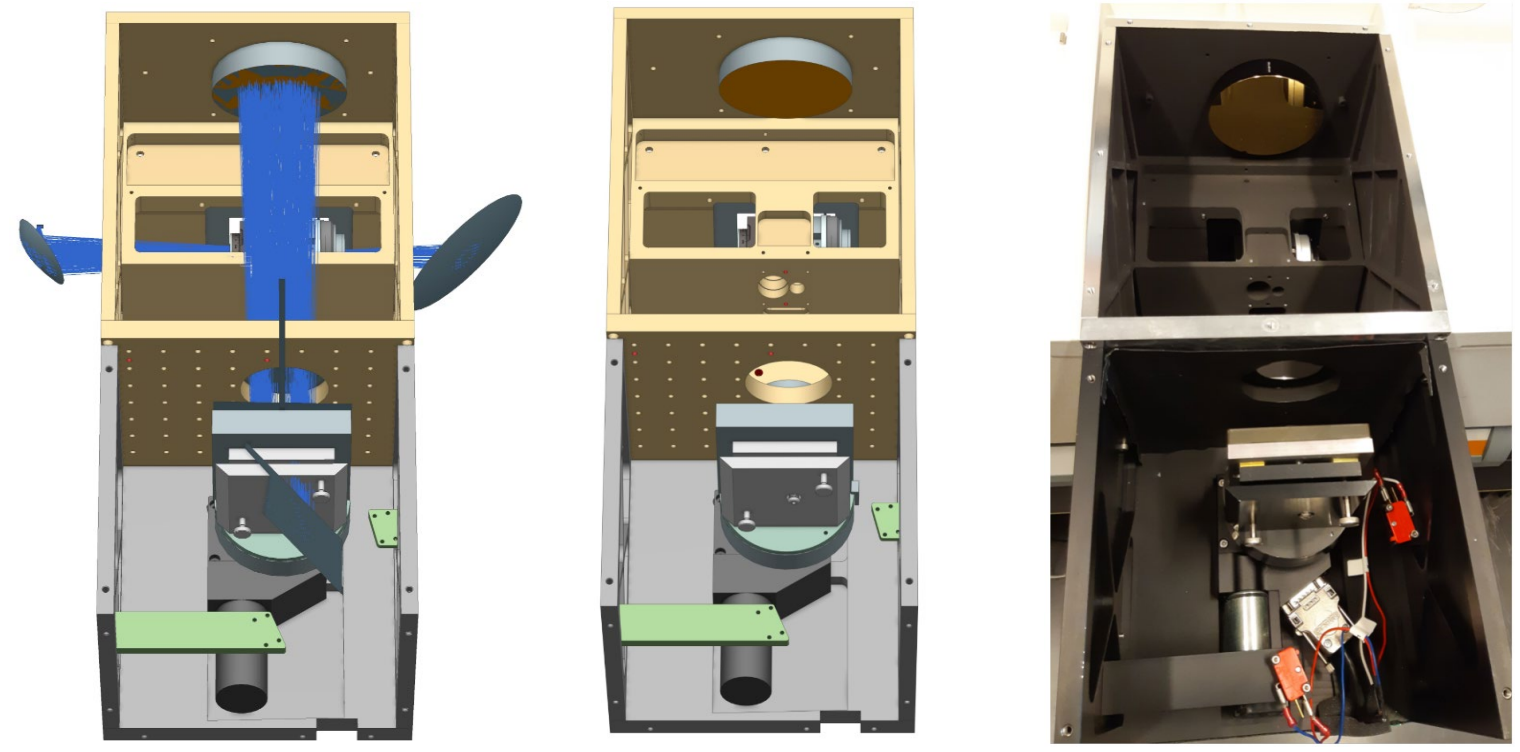

Figure 11. The CAD design of the FTS extension using the reference mirror is shown (left and middle). The real built setup is shown on the right. 
The mechanical structure containing all relay optics was mounted inside the FTS measurement compartment, while the reference mirror or the immersed grating could be mounted in a mechanical extension ('balcony') outside of the FTS volume. The complete setup was closed and purged by dry filtered air from outside. Since the setup was significantly larger than the original FTS, additional purging time was needed. At least 2 hours purging was maintained when switching between the reference mirror and the immersed grating. Both the reference mirror and the immersed grating had to be aligned in tip and tilt to avoid vignetting, which would affect the measurements.

The reference mirror measurements were used to monitor FTS stability and the repeatability of the mechanism used. The stability of the signal using the reference mirror was below $0.5 \%$ and the repeatability of the mechanisms (reference mirror and polarizer rotation stages) were less than $0.1 \%$ in terms of signal variation. Also, the test sequence was designed so that the immersed grating measurements were always embedded between two reference mirror measurements (before and after) to monitor the stability of the FTS.

The throughput measurements were done first without any polarization. In Figure 12 the absolute Ge immersed grating throughput is shown for random input polarization for the complete 2.9-5.3 $\mu \mathrm{m}$ wavelength range. The FTS has a small instantaneous wavelength coverage compared to the free spectral range of the grating, so the rotation of the immersed grating is necessary. The real grating efficiency can be determined by looking at the cover curve of the peaks of the individual diffraction orders, when the immersed grating is tilted to cover the complete free spectral range.

In the present measurement sequence, the immersed grating is rotated by one and (close to the peaks) half degree intervals to scan the free spectral range provided by the grating. The peak throughput is between $62 \%$ and $72 \%$, highest in the 3.1-3.3 $\mu \mathrm{m}$ range and lowest in the 4.1-4.6 $\mu \mathrm{m}$ range (Figure 13). A grating rotation between -1.5 and -2.5 degrees provided the highest throughput in most of the cases, corresponding to the near Littrow configuration.

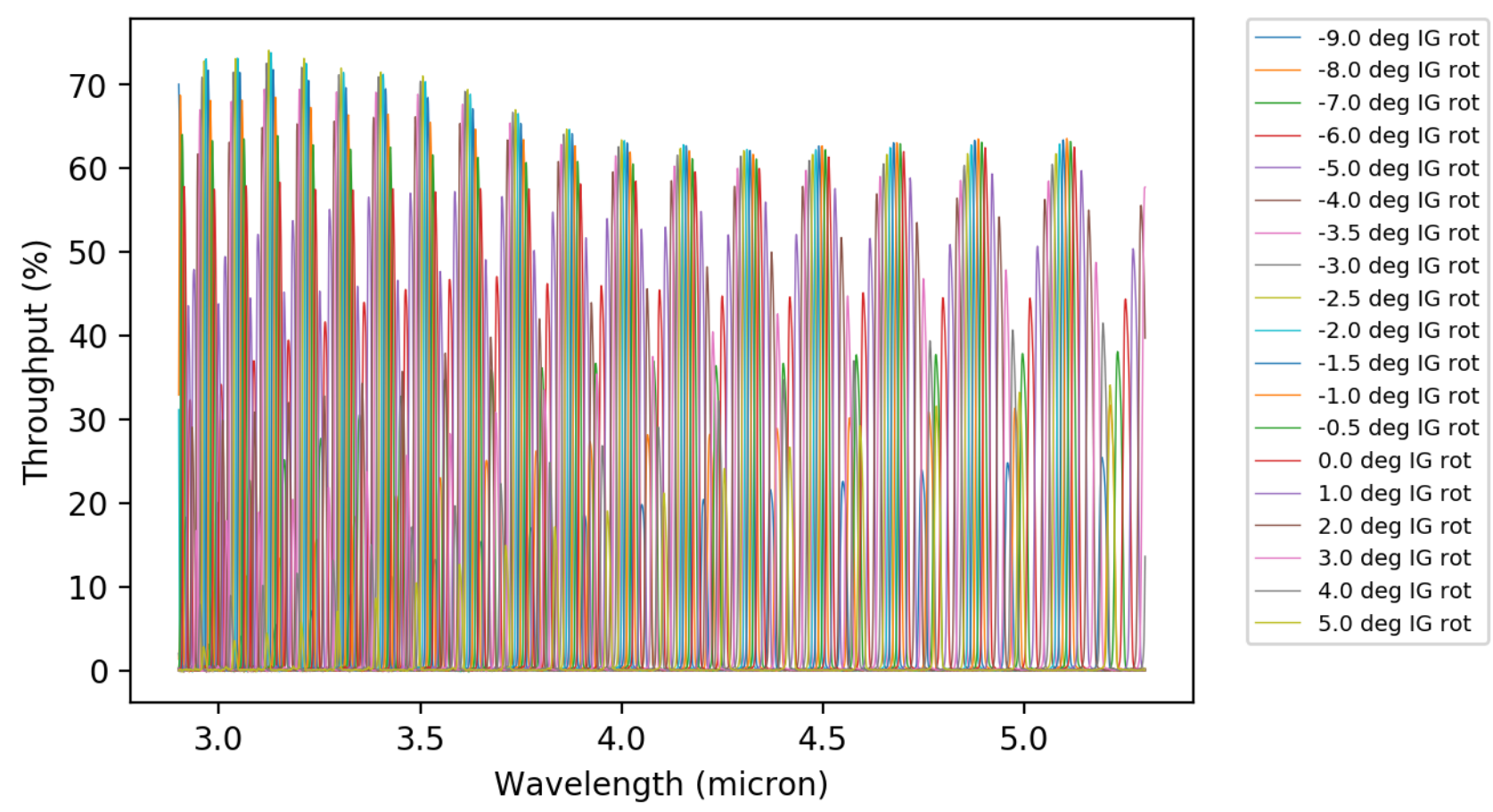

Figure 12. The Ge immersed grating throughput is shown for random input polarization for the complete 2.9-5.3 $\mu \mathrm{m}$ wavelength range. The immersed grating is rotated by one degree and close to the peaks 0.5 degree intervals to scan all the individual orders provided by the grating. 

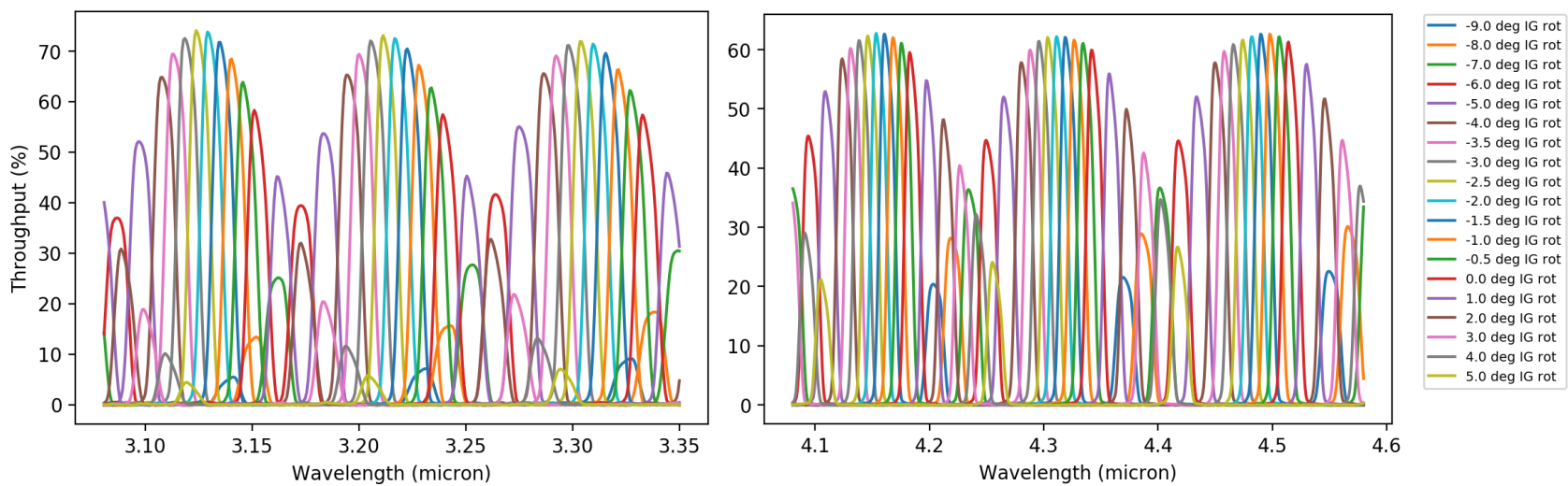

Figure 13. Same as in Figure 12, but smaller wavelength ranges are shown, where the maximum (left) and minimum (right) throughput occur.

After the non-polarized tests, the wire-grid polarizer was mounted in the relay optics in the input beam. The reference mirror and immersed grating measurements were repeated. The reference 0 degree of the motorized rotation stage was aligned such that the direction of the linear polarization projected on the grating surface was perpendicular to the grating lines (90 degree rotation was parallel with the grating lines). Measurements were taken using polarization directions of 0 , $30,75,90,120$ and 165 degrees.

In the following two figures, the absolute Ge immersed grating throughput is shown for the complete 2.9-5.3 $\mu \mathrm{m}$ wavelength range, when the input is linearly polarized and the polarization direction is rotated by 30 degrees (Figure 14) and 120 degrees (Figure 15). 30 degree polarization orientation resulted the highest and 120 degrees the lowest overall throughput. In the latter case there is a valley in the throughput curve centred around 4 microns.

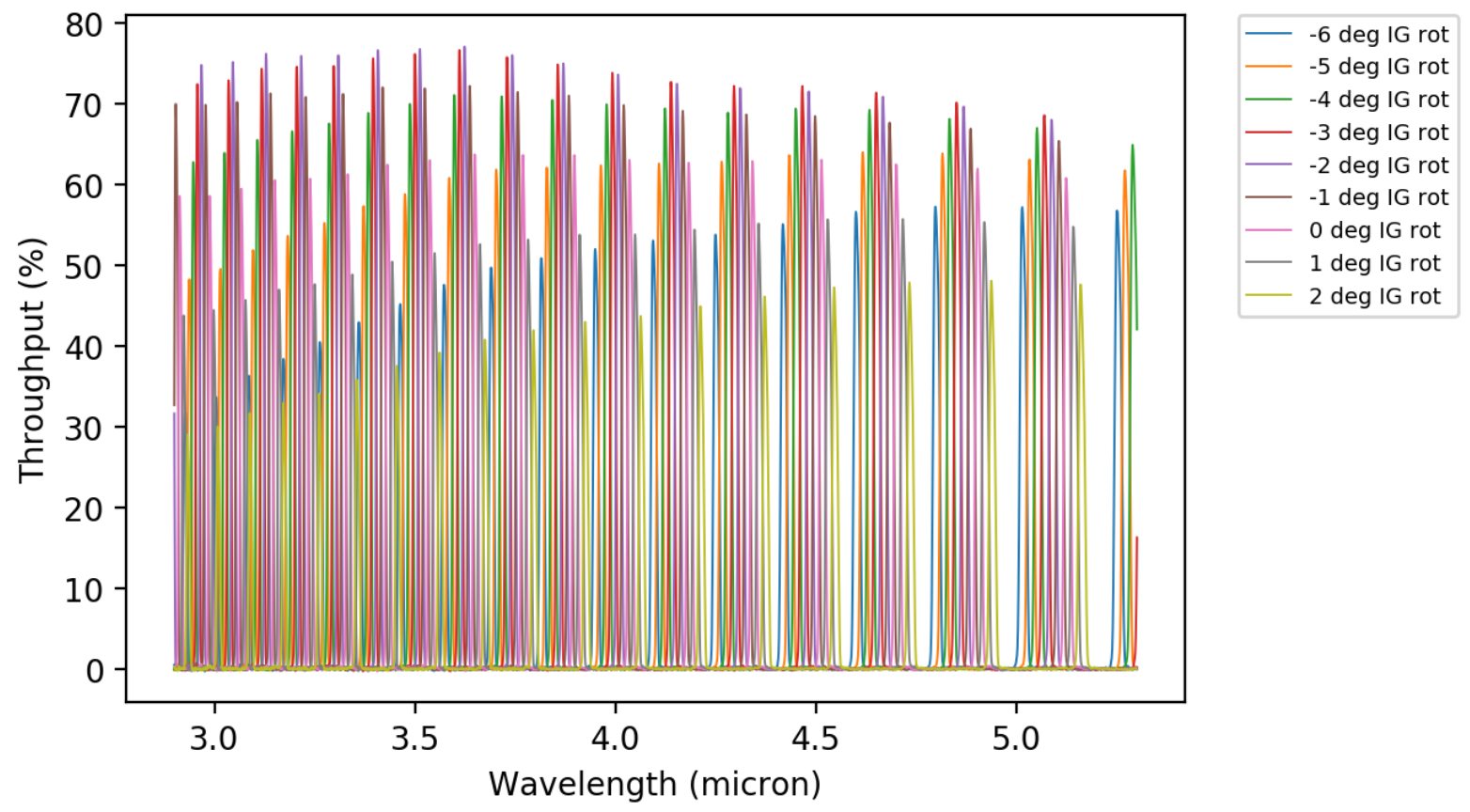

Figure 14. Same as in Figure 12 but the input is linearly polarized and the polarization direction is rotated by 30 degrees (90 degree rotation is parallel with the grating lines). The present polarization orientation resulted the highest overall throughput. 


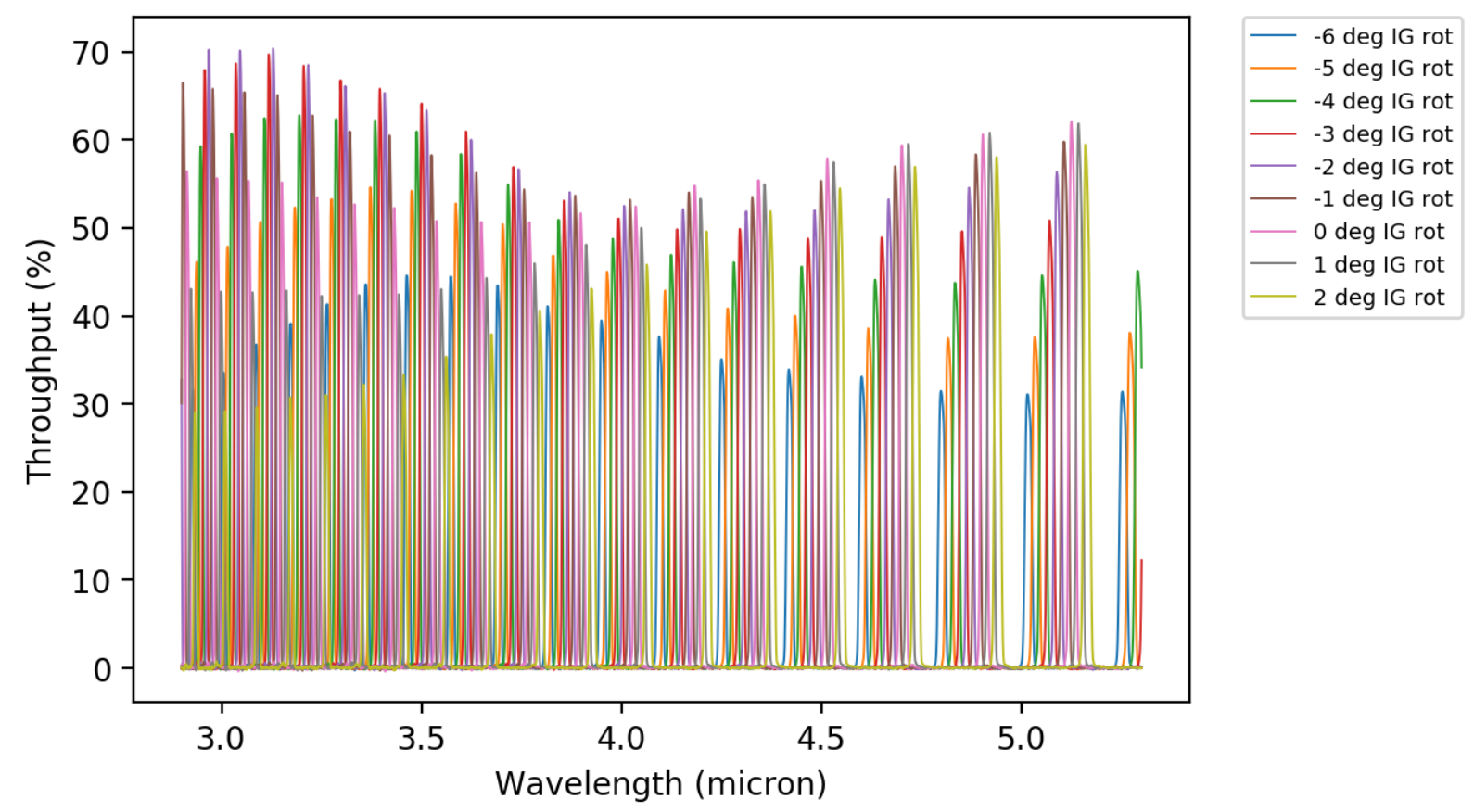

Figure 15. Same as in Figure 12 but the input is linearly polarized and the polarization direction is rotated by 120 degrees (90 degree rotation was parallel with the grating lines). The present polarization orientation resulted the lowest overall throughput.

In the following the throughput is depicted as a function of polarization direction using linearly polarized input. The sinusoidal curve is clearly visible alongside with the varying efficiency with changing wavelength. One can observe that the highest throughput occurs around 30 degree and the lowest is around 120 degrees. When the linearly polarized light is perpendicular to ( 0 degree rotation) or parallel with ( 90 degree rotation), the throughput is actually very similar. We will investigate further why such throughput dependency on input polarization direction is observed.
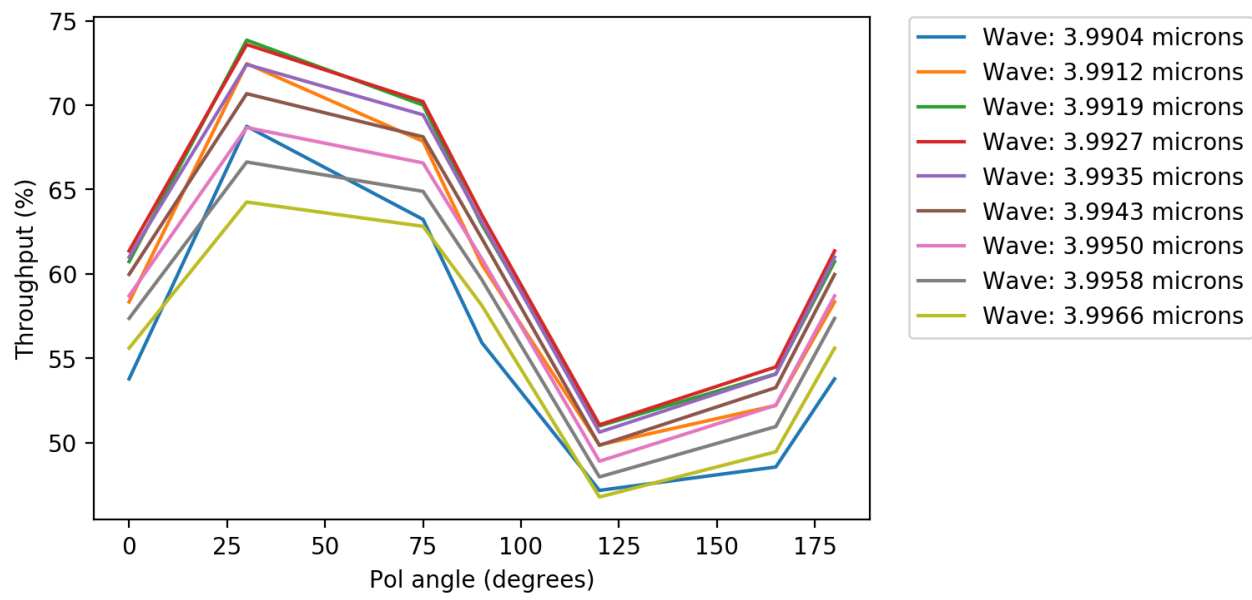

Figure 16. The throughput is shown as a function of polarization direction using linearly polarized input. The sinusoidal curve is clearly visible alongside with the varying efficiency with changing wavelength. The immersed rating is rotated -3 degrees for this particular measurement. 


\section{Comparison of the cascade laser and FTS based measurements}

By comparing the MIRcat cascade laser and FTS based measurements one can conclude that the results are similar but there are some smaller deviations between them. Firstly, the MIRcat measurements show a slightly higher (2-3\%) throughput, secondly, the diffraction order peaks are slightly shifted with respect to each other. Finally, the shape of these peaks are also distinct. On the other hand both measurements show the same $\sim 18-20 \%$ throughput difference between perpendicular polarizations.

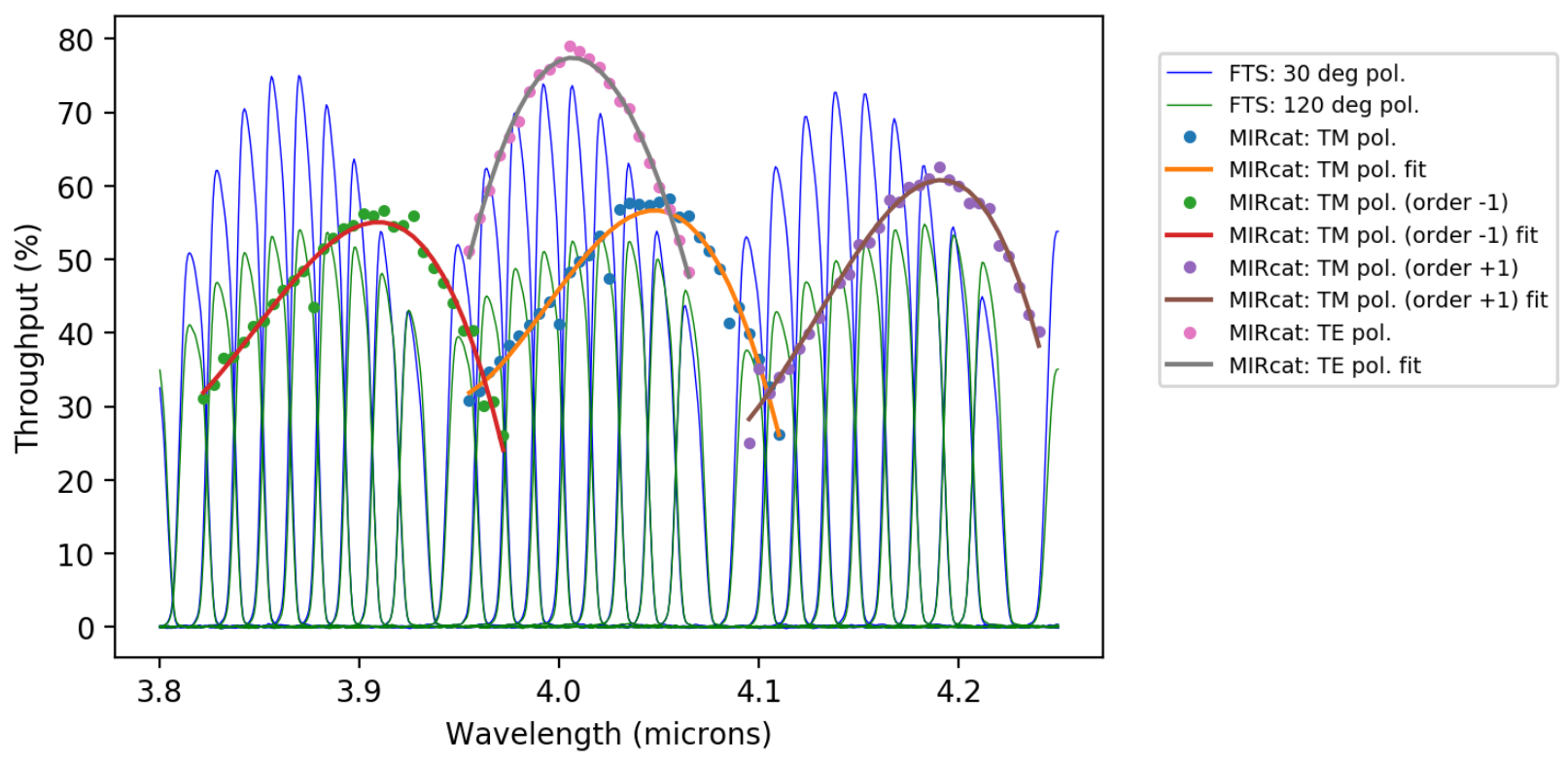

Figure 17. The throughput is shown from the two measurements. The MIRcat cascade laser based measurement shows a slightly higher throughput and the peaks are displaced with respect to the FTS measurements.

The few percent throughput difference between the two measurements come from the FTS or the cascade laser setup inaccuracies. From the FTS side, the inaccuracy of the reference mirror reflectivity can play a role. We also observe a slightly higher mean throughput, when the spectral resolution of the FTS is increased from $2 \mathrm{~cm}^{-1}$ to $0.25 \mathrm{~cm}^{-1}$ (Figure 18). The selected FTS spectral resolution for the measurement campaign was $1 \mathrm{~cm}^{-1}$ because for higher spectral resolution the observed noise increased significantly.

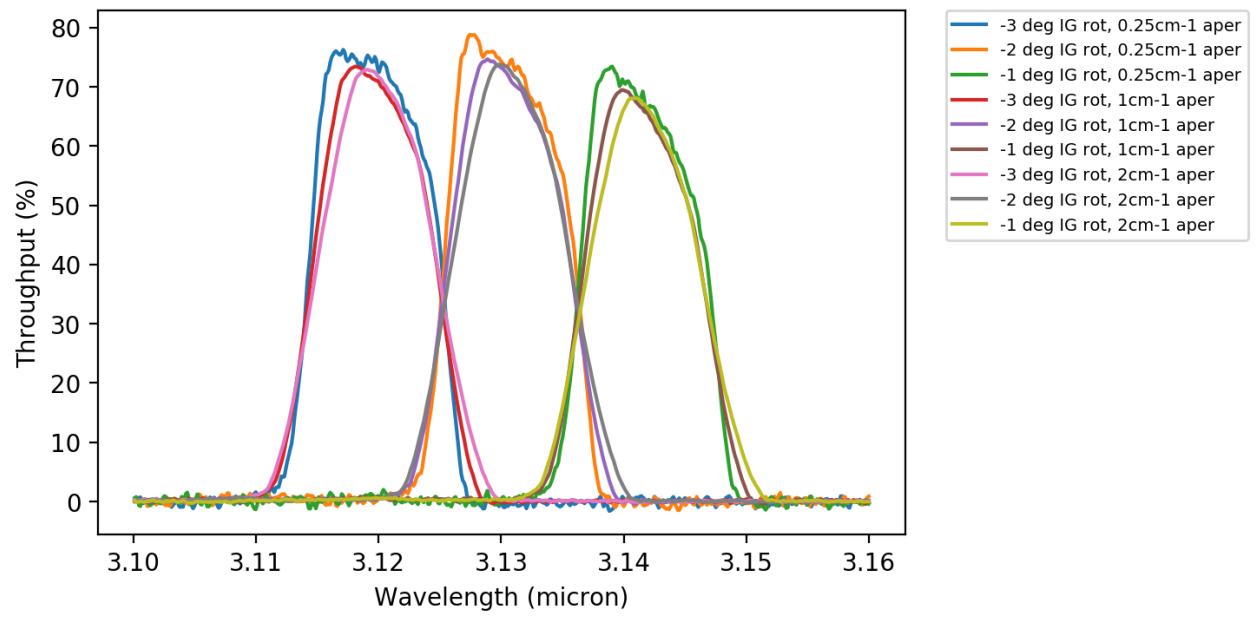

Figure 18. The absolute throughput is shown using different FTS spectral resolutions $\left(0.25 \mathrm{~cm}^{-1}, 1 \mathrm{~cm}^{-1}\right.$, and $\left.2 \mathrm{~cm}^{-1}\right)$. The highest spectral resolution $\left(0.25 \mathrm{~cm}^{-1}\right)$ measurement has significant noise, so it was decided to use $1 \mathrm{~cm}^{-1}$ resolution for most of the FTS measurements. 


\section{CONCLUSIONS}

We presented the manufacturing and optical verification of the germanium immersed grating for the $\mathrm{L} / \mathrm{M}$ band high resolution spectrograph (LMS). The LMS is one of the science subsystems of the Mid-infrared ELT Imager and Spectrograph, METIS. The immersed grating has very demanding requirement specifications: $<100 \mathrm{~nm}$ RMS transmitted Wave Front Error (WFE) after double pass, and $>70 \%$ peak throughput in all orders within the 2.9-5.3 $\mu \mathrm{m}$ wavelength range over the pupil. The grating has a period of $18.2 \mu \mathrm{m}$, a sawtooth groove profile with 89.6 degrees apex angle and a grating area of $150 \mathrm{~mm} \times 60 \mathrm{~mm}$.

The germanium immersed grating was produced by Canon's high precision mechanical cutting technology. The interferometric tests show that the immersed grating has very low surface form error in the entrance/exit surface. Additionally, the WFE is excellent when the grating surface is measured from air side in double pass. Not taking into account any material based aberrations (birefringence or refractive index inhomogeneity), the calculated double pass RMS WFE of the immersed grating is $64.6 \mathrm{~nm}$, which is way below the $100 \mathrm{~nm}$ requirement specification.

Two different measurements (based on cascade laser and Fourier Transform Spectrometer, respectively) were done for throughput verification. Both measurements show the same $\sim 18-20 \%$ throughput difference between perpendicular polarizations. The cascade laser measurements show a slightly higher (2-3\%) throughput both for TE and TM polarization. Looking at the FTS measurements results for randomly polarized input, the peak throughput is between $62 \%$ and $72 \%$; thus the manufactured immersed grating satisfies the $>70$ peak throughput requirement, but not in the complete LM wavelength range.

In the next stages of this work we will update the transmission budget of the instrument and investigate how the lower throughput, observed in the longer wavelength ranges, would affect the science operations of METIS. We will study the throughput dependency on linear polarization direction in the case of the FTS measurements. We will use the optical model to calculate the polarization response of the FTS test system. Finally we are also considering stray light measurements in spatial and spectral directions. The lower throughput may mean that there is more straylight than expected and so the intensity of the stray light and also the identification of possible ghosts and satellites should be considered by e.g. dedicated Bidirectional Reflection Distribution Function (BRDF) measurements.

\section{ACKNOWLEDGEMENTS}

Thank you for Mr. Takashi Sukegawa and the Canon team for their contribution and providing the information on the immersed grating manufacturing and optical verification.

\section{REFERENCES}

[1] Bizenberger, P. et al., 'METIS: Design of the Imager Sub-System,' Proc. SPIE 11447, Paper 11447-380 (2020).

[2] Absil, O. et al., 'Towards METIS final design for rocky planet imaging on the ELT,' Proc. SPIE 11447, Paper 11447-390 (2020).

[3] Agocs, T. et al., 'Preliminary optical design for the common fore optics of METIS,' Proc. SPIE 9908, Paper 99089Q (2016).

[4] Labadie, L. et al., 'The warm calibration unit of METIS: Optical design and principle of operation,' Proc SPIE 11447, Paper 11447-348 (2020).

[5] T. Sukegawa, T. Suzuki and T. Kitamura, 'Astronomical large Ge immersion grating by CANON,' Proc. SPIE 9912, Paper 99122V (2016).

[6] P. J. Kuzmenko et al., 'Materials and fabrication issues for large machined germanium immersion gratings,' Proc. SPIE 6273, Paper 62732W (2006) 